\title{
How do general practitioners manage eye disease in the community?
}

\section{P J MCDONNELL}

From the Department of Ophthalmology, St Thomas's Hospital, London

SUMMARY A survey of the management of eye disease in the community was carried out in two general practices over a three-month period. During this time there were 238 consultations by patients with ocular symptoms, making up $2.3 \%$ of all consultations and giving an annual consultation rate for eye disease of 66 per 1000 persons at risk. The four commonest diagnoses were bacterial conjunctivitis, allergic conjunctivitis, meibomian cyst, and blepharitis, and these accounted for more than $70 \%$ of the consultations. A variety of topical and systemic treatments were used, with topical chloramphenicol prescribed in 55\% of consultations. Referral to a hospital eye department resulted from 35 consultations, giving a referral rate of $15 \%$ of all consultations.

There are few detailed studies assessing the way in which general practitioners manage eye disease in the community. The most comprehensive data in this country on prevalence of eye disease come from the morbidity statistics of the Royal College of General Practitioners. ${ }^{1}$ However, their classification of eye disease is into broad categories: for instance there is no differentiation between the two common conditions of bacterial conjunctivitis and allergic conjunctivitis, and there is no assessment of the management of eye diseases by general practitioners.

Ophthalmologists are increasingly aware of the importance of primary ophthalmic care in the community. An improvement in both undergraduate and postgraduate teaching of ophthalmology is vital to help the general practitioner to become more confident in the diagnosis and management of the wide range of eye diseases found in general practice.

An assessment of how general practitioners treat eye disease in the community at present might help in planning future teaching. This survey was carried out over three months in two general practices in South London. The aims of the survey were to determine the prevalence of eye diseases in the two general practices and to determine how these diseases were treated by the general practitioners.

Correspondence to Mr P J McDonnell, FRCS, South Wing Eye Department, St Thomas's Hospital, Lambeth Palace Road, London SE1 7EH.

\section{Subjects and methods}

The survey was carried out for the three months July 1986 to September 1986 in two general practices. One practice consisted of four general practitioners in Clapham, South London, with a practice population of 9521 patients. The other practice consisted of three general practitioners in Streatham, South London, with a practice population of 4880 patients, making a total study population of 14401 . Analysis of the practice populations by age shows that $87 \%$ were under $65,7 \%$ were 65 to 74 , and $6 \%$ were 75 or older. This breakdown reflects the nature of the two practice areas, with fewer old people than the national average and a large number of working people, both single and married, in the 25-44 age group.

All consultations of patients with ocular symptoms were with general practitioners. A special form was used to record the presenting symptoms, the diagnosis made by the general practitioner, and the subsequent management including details of drug treatment and any referral.

\section{Results}

During the period of the survey there were 238 consultations by 224 patients with ocular symptoms, representing $2 \cdot 3 \%$ of the total number of medical consultations, which was 10182 . This gives a consul- 


\section{Discussion}

Studies of patients with ocular symptoms in general practice have tended to limit their inquiries to the prevalence of eye disease. The most extensive survey in this country is that organised by the Royal College of General Practitioners. In the report on 1981-2 morbidity statistics from general practice ${ }^{1}$ the results from a year-long survey gave an annual consultation rate for patients with eye disease of 70.1 per 1000 , representing $2.06 \%$ of all medical consultations. More recently an ophthalmologist carried out a three-month study at a community health centre and found an annual consultation rate of 57 per 1000 , representing $2 \cdot 7 \%$ of all consultations. ${ }^{2}$ These figures are comparable with this three-month study, where the consultation rate was 66 per 1000 , representing $2 \cdot 3 \%$ of all consultations.

The Royal College of General Practitioners study is of more limited value in reviewing the range of conditions seen by general practitioners, as there are only 12 categories of diagnoses, and common conditions such as bacterial conjunctivitis and allergic conjunctivitis are included under the one heading of conjunctivitis. The three commonest diagnoses were conjunctivitis, meibomian cyst, and blepharitis, which are the same three as in the present study.

The study by Dart, ${ }^{2}$ which took place between the months of May and August, has allergic conjunctivitis as the commonest diagnosis, accounting for $21 \%$ of cases, while bacterial conjunctivitis accounts for only $4.7 \%$ of patients seen by the ophthalmologist in the community. This contrasts with the present study, which also took place in the summer, where bacterial conjunctivitis accounts for $44 \%$ of cases and allergic conjunctivitis accounts for $15 \%$ of cases. Interestingly in Dart's study, when patients saw only the general practitioner, the diagnosis of bacterial conjunctivitis was more likely, being made in $39 \%$ of cases. It could be that general practitioners overdiagnose bacterial conjunctivitis at the expense of other diagnoses: the ophthalmologist had a slit-lamp in the surgery, which made more detailed examination possible. It may also be that the patients seen by the ophthalmologist were a selected group, as some patients who saw a general practitioner then declined a further visit to see the ophthalmologist.

As both Dart's study and the present study were carried out during the summer months, the prevalence of allergic conjunctivitis is likely to have been higher than at other times of the year and thus possibly have influenced the overall consultation rates. However, extrapolation of the three-month results from both studies gives annual consultation rates very similar to those reported in the Royal College survey.
The chronic conditions of glaucoma and cataract were infrequently diagnosed in the present study only one case of each in the three month period. This contrasts with the Royal College study, where cataract and glaucoma together accounted for $7 \cdot 6 \%$ of diagnoses. There are two factors which may explain this discrepancy. The practice areas studied had a lower percentage of patients over $64(13 \%)$ than the national average of $15 \%$. In addition there is easy access to hospital eye casualty and clinic services in the area, which may reduce the number of consultations with general practitioners for cataract and glaucoma.

Only two recent studies mention the treatment used by general practitioners in the management of eye diseases. A year-long Australian study found that chloramphenicol was the commonest antibiotic used for infective problems, but no figures are given. ${ }^{3}$ That study also found that topical steroids were occasionally prescribed, but again no figures are given. A recent postal survey carried out in this country asked general practitioners about their management of the red eye. ${ }^{4}$ Topical chloramphenicol was used by $93 \%$ of the respondents to treat infective conjunctivitis, but for non-specific conjunctivitis $10 \%$ chose a steroid-containing preparation. This survey, however, asked only about prescribing intentions and was not a retrospective study of patient management. The general practitioners in the present study treated patients with a fairly limited range of drugs. Chloramphenicol was the most popular antibiotic used topically.

The most frequent treatment for allergic conjunctivitis was sodium cromoglycate. In view of a recent editorial ${ }^{5}$ and the study by Lavin and Rose ${ }^{6}$ it is interesting to note that $2.9 \%$ of patients were treated with topical steroids. These patients were mainly suffering from allergic conjunctivitis that had not responded to topical sodium cromoglycate. No patients were treated with combined steroid and antibiotic prescriptions.

The referral rate for patients in the study was $15 \%$ of all consultations and was for a wide range of diagnoses. The Royal College survey does not record the referral rate for patients with ocular symptoms, but the overall referral rate for all diseases and conditions is $16.5 \%$, which is comparable to the rate in this survey.

This study has shown that general practitioners successfully manage a wide range of eye diseases in the community. The diseases they see may be divided into two groups: common external eye conditions such as conjunctivitis and meibomian cyst, which are not sight threatening and are relatively easy to treat in the community, and secondly rare but sight threatening conditions such as retinal detachment 
and glaucoma, which must be referred to a hospital eye department.

Patients were treated by their general practitioners with a limited range of drugs the majority of which could be used safely in the community. Teaching of medical students and update of general practitioners should do more to emphasise the two broad groups of patients with eye disease in the community and to encourage the use of a limited range of safe topical medications in the treatment of patients with eye disease in general practice.

I thank Drs P K Madon, A J Surawy, C J Peach, and J H McDonnell and the staff of the Surgery, Battersea Rise, London SW11, and Drs J C Austen, T Foster, and S J Savage and the staff of the Surgery, Baldry Gardens, Streatham, London SW16, for their help with the study.

\section{References}

1 Royal College of General Practitioners, Office of Population Censuses and Surveys and Department of Health and Social Security. Morbidity statistics from general practice 1981-82. London: HMSO, 1986: Series MB5 no.1.

2 Dart JKG. Eye disease at a community health centre. Br Med J 1986; 293: 1477-80.

3 Rowe IL. Eye conditions, prevalence and treatment: from the Australian morbidity survey. Aust Fam Physician 1973; 2: 513-4.

4 Wilson A. The red eye: a general practice survey. $J R$ Coll Gen Pract 1987; 37: 62-4.

5 Roberts D St C. Steroids, the eye, and general practitioners. (Editorial.) Br Med J 1986; 292: 1414-5.

6 Lavin MJ, Rose GE. Use of steroid eye drops in general practice. Br Med J 1986; 292: 1448-50.

Accepted for publication 13 July 1987. 